\title{
Autoerythrocyte sensitization (Gardner-Diamond syndrome) in men: a case report and review of the literature
}

\author{
A. Ingber, J. Alcalay and E.J. Feuerman \\ Department of Dermatology, Beilinson Medical Center, Petach-Tiqva, Sackler School of Medicine, Tel-Aviv \\ University, Israel
}

\begin{abstract}
Summary: Autoerythrocyte sensitization (AES) is a rare syndrome particularly among males. A young male with typical AES is herewith described. We review all seven published cases of AES in men for which details are available and compare the age of onset, somatic manifestations, associated diseases and psychiatric disturbances between the sexes. We emphasize that the criteria essential for a diagnosis of AES should include typical clinical presentation, longstanding clinical history and a positive skin test with the patient's blood. Because of the rarity of AES there is a lack of awareness regarding it among many doctors.
\end{abstract}

\section{Introduction}

Autoerythrocyte sensitization (AES) is a rare disorder described mainly in women. The appearance of AES in men is very rare and only 6 cases have been previously published. We describe a young male with AES and compare the syndrome in men and women.

\section{Case report}

An 18 year old white male was referred because of recurrent painful ecchymoses in his legs which had appeared for the first time when he was 11 years old. He had since suffered several painful ecchymoses every year which appeared only after trauma to his legs. This phenomenon was investigated in several hospitals, but no diagnosis was made. He was treated surgically by evacuating the haematomata followed by complete bed rest for several days. The post-traumatic ecchymoses appeared exclusively in his legs and no other part of the body was involved. The patient described that a few minutes after the trauma he began to feel a burning sensation at the injured site. This sensation was always followed by left frontal headache and within minutes of this sensation the skin at the injured site reddened and a bruise appeared. The swelling increased in size and resembled 'half an apple'. The bruise was painful and tender to the touch for several days. The patient had no history of bleeding tendencies and no psychiatric background.

Correspondence: A. Ingber, M.D., Department of Dermatology, Beilinson Medical Center, Petach-Tiqva, 49100, Israel

Accepted: 5 March 1985
Physical examination revealed only several iatrogenic old scars in both legs and a residual haematoma in the right leg. Laboratory investigations proved normal or negative. These included full blood count, platelet count, prothrombin time, partial thromboplastin time, factor VIII, factor 13, clot retraction, clotting time, bleeding time (Ivy), complement, C-1 esterase inhibitor, latex agglutination, antinuclear and anti-DNA antibodies, immunoelectrophoresis. Psychiatric evaluation was unremarkable.

Intradermal injection of $0.1 \mathrm{ml}$ of autologous blood caused an immediate burning sensation at the injection site. Two hours after injection an erythematous macular lesion appeared. The lesion was $2 \mathrm{~cm}$ in diameter, oval shaped and had clear margins distinguishing it clearly from the surrounding area (Figure 1). Intradermal injection of $0.1 \mathrm{ml}$ of saline caused no reaction. These findings were accompanied by left frontal headache.

\section{Discussion}

Autoerythrocyte sensitization was first described by Gardner \& Diamond (1955) in four women. Since the original report more than 100 patients with AES have been reported, most of them women (Campbell et al., 1983). In the majority of the patients a psychogenic basis was found (Agle \& Ratnoff, 1962). In 1971 a young male, the first with psychosomatic purpura, was described (Lababidi \& Friedman, 1971). Since then 5 more cases of the syndrome have been reported in men (Kurczynski et al., 1973; Goldsmith et al., 1976; 


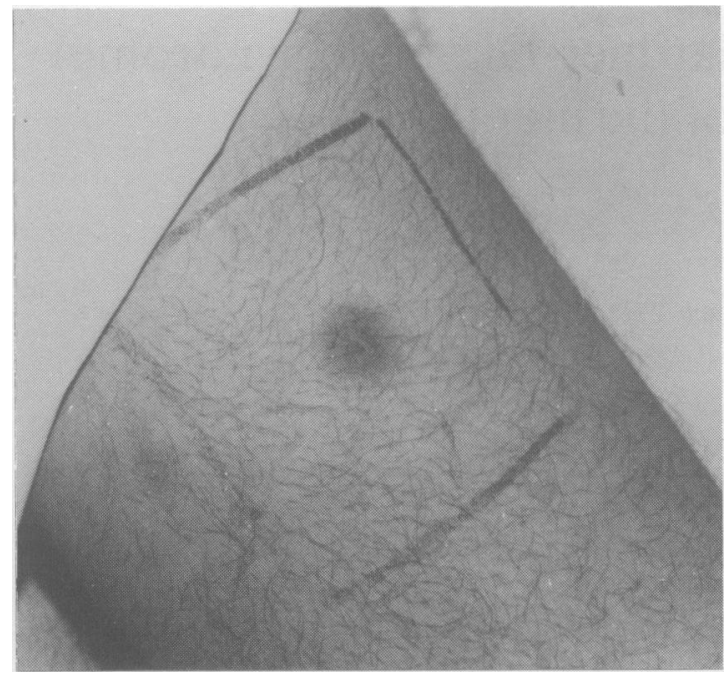

Figure 1 An erythematous macular lesion, $2 \mathrm{~cm}$ in diameter, on the left thigh, appeared $2 \mathrm{~h}$ after the injection of $0.1 \mathrm{ml}$ of autologous blood, intradermally.

Shustik, 1977; Akman et al., 1978; Glowinski et al., 1981). Although Ratnoff (1980) in his extensive review of AES mentioned an additional 3 cases of AES in males, we cannot consider these cases here because they were not described in detail.

Table I summarizes all the published cases of AES in men. The onset of AES in men occurs in the second or third decade of life. Except for patient no. 5 all the men had the first episode of bruising when they were less than 21 years old. Although recently Campbell et al. (1983) reported 6 cases of AES in girls, the majority of women with AES are older than 20 at the onset (Ratnoff, 1980).

The ecchymoses in AES are most commonly localized in the extremities including the hands (Hersle \& Mobacken, 1969), but ecchymoses in other sites such as abdomen, chest, breasts, shoulders, neck and face have been reported as well (Ratnoff, 1980). Most of the men with AES had bruises in the extremities, but patients no. 2 and no. 4 also had ecchymoses on the shoulders and abdomen. The lesion can appear shortly after an injury or surgical procedure (Gardner \& Diamond, 1955) or spontaneously (Ratdnoff, 1980). All the men had spontaneous bruises except our patient who suffered exclusively from post-traumatic ecchymoses. Although Ratnoff emphasizes that the significance of physical injury as an inciting event must be tempered in the light of the surrounding circumstances, we could not find any emotional stresses when symptoms first appeared in our patient. The lesions usually appear on both sides, but occasionally just on one side of the body. We found no difference in the site of bruising nor in the duration of the illness between

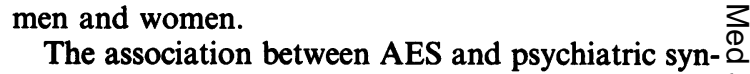
dromes has been recognized and the term 'psychogenic $\subseteq$ purpura' proposed. Ratnoff described five compon- $\overrightarrow{\bar{B}}$ ents of the psychological makeup of his patientsincluding hysteria, masochism, depression, anxiety and inability to deal with hostile feelings. Four of the $\frac{\bar{\omega}}{\bar{\omega}}$ male patients (no. 1, 2, 4, 5) had a psychiatric back- $\overrightarrow{\widehat{\phi}}$ ground similar to the emotional problems described in $\bigcirc$ the women. Ou= patient showed no psychiatric abnor- $\mathcal{E}$ mality. Ratnoff reported that three of the five adult $\vec{\circ}$ male patients previously described by other authors were impotent in contrast with only one of the seven $\vec{\sigma}$ reviewed here. We think that the stereotypic person- $\frac{}{0}$ ality structure described in women with AES is only partly found in men.

Patients with AES present a variety of somatic $\stackrel{-}{-}$ complaints and associated diseases. These include $\vec{\bullet}$ gastrointestinal bleeding, epistaxis or other non- $\stackrel{\infty}{\infty}$ cutaneous bleeding (Khan \& Cash, 1970; McDuffie \& McGuire, 1965; Stocker et al., 1977). Symptomso referable to the nervous system are frequent and ${ }_{-}$ episodic abdominal pain with or without attacks of $c s$ nausea or vomiting have been repeatedly described $\mathbb{D}$ (Lindahl, 1977; Ogston et al., 1971; DiGrande, 1972; Waldorf \& Lipkin, 1968). Our patient had unilateral frontal headache but had no other somatic manifest 8 tion associated with the syndrome. The relationship $\overrightarrow{0}$ between the associated disorders and AES is net or known. We found no difference between the somatic manifestations in men and women.

Gardner \& Diamond (1955) evoked the typical lesions by intradermal injection of erythrocytic stroma. Although the intradermal test conditions $\Phi$

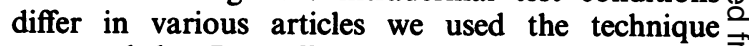
proposed by Ratnoff (1980). In our patient the $\overrightarrow{\bar{O}}$ negative result at the control injection site and the $\frac{3}{5}$ appearance of a $2 \mathrm{~cm}$ lesion at the site of blood injection together with the appearance of frontal headache was sufficient for the diagnosis. In all except $\frac{}{3}$ one of the men (no. 6) similar tests utilizing autologous blood were performed, four of them being considered 3 . positive. We believe that a positive skin test with autologous blood or erythrocytic stroma in a man suspected to have AES, is necessary and sufficient for 0 the diagnosis. Except for the outstanding feature of cryofibrinogenaemia in patient no. 2 , in all the other $\frac{}{2}$ men the results of laboratory studies revealed nothing remarkable. This is consistent with the findings in of women.

Ratnoff (1980) reported that AES in men occurs in $N$ $5 \%$ of all AES cases. In our opinion the percentage of males with AES is lower because since Ratnoff's report only one additional male has been recorded. AES in men and women has common characteristics, $\stackrel{\odot}{\oplus}$ although the average age of the first episode is lower in men. 


\section{References}

AGLE, D.P. \& RATNOFF, O.D. (1962). Purpura as a psychosomatic entity. A psychiatric study of autoerythrocyte sensitization. Archives of Internal Medicine, 109, 685.

AKMAN, N., AKTUGLU, G., EKER, E. \& CIVGA, S. (1978). A male case of autoerythrocyte sensitization syndrome due to hemoglobin sensitivity. Acta Haematologica, 59, 250.

CAMPBELL, A.N., FREEDMAN, M.H. \& MCCLURE, P.D. (1983). Autoerythrocyte sensitization. Journal of Pediatrics, 103, 157.

DIGRANDE, E. (1972). Psychogenic purpura: platelet factor 3 deficiency. Archives of Dermatology, 104, 444.

GARDNER, F.H. \& DIAMOND, L.K. (1955). Autoerythrocyte sensitization. A form of purpura producing painful bruising following autosensitization to red blood cells in certain women. Blood, 10, 675 .

GLOWINSKI, J., KOUBI, R., MAY, V. \& LEIBOWITCH, M.L. (1981). Syndrome de Gardner et Diamond chez un homme essai de traitment par les betabloquants. La Nouvelle Presse Médicale, 2710, 2369.

GOLDSMITH, G., MUIR, W.A. \& AGLE, D.P. (1976). Autoerythrocyte sensitization in a man with Ehlers-Danlos; further evidence for reliability of intracutaneous autologous blood injection in diagnosis (abstract). Clinical Research, 24, 309a.

HERSLE, K. \& MOBACKEN, H. (1969). Autoerythrocyte sensitization syndrome (painful bruising syndrome). Report of two cases and review of the literature. British Journal of Dermatology, 81, 574.
KHAN, S.A. \& CASH, J.D. (1970). Autoerythrocyte sensitization syndrome. Scottish Medical Journal, 15, 248.

KURCZYNSKI, E.M., CASSIDY, J.T. \& HEYN. R.M. (1973). Autoerythrocyte sensitization in a young boy (letter). Lancet, i, 424.

LABABIDI, Z. \& FRIEDMAN, R. (1971). Psychosomatic purpura. Missouri Medicine, 68, 386.

LINDHAL, M.W. (1977). Psychogenic purpura: report of a case. Psychosomatic Medicine, 39, 358

McDUFFIE, E.C. \& McGUIRE, F.L. (1965). Clinical and $\vec{\circ}$ psychological patterns in autoerythrocyte sensitivity. Annals of Internal Medicine, 63, 255.

OGSTON, D., OGSTON, W.D. \& BENNET, N.B. (1971), Psychogenic purpura. British Medical Journal, 1, 30.

RATNOFF, O.D. (1980). The psychogenic purpuras: A review 3 of autoerythrocyte sensitization, autosensitization to DNA, 'Hysterical' and factitial bleeding and the religious stigmata. Seminars in Hematology, 117, 192.

SHUSTIK, C. (1977). Gardner-Diamond syndrome in a man. Archives of Internal Medicine, 137, 1621.

STOCKER, W.W., MCINTYRE, D.R. \& CLENDENNING, W.E. (1977). Psychogenic purpura. Archives of Dermatology, 113, 606.

WALDORF, D.S. \& LIPKIN, G. (1968). Sensitization to erythrocytes. A distinctive syndrome. Journal of the American Medical Association, 203, 597. 